\title{
Exact Solution of Riccati Fractional Differential Equation
}

\author{
Khaled Jaber*, Shadi Al-Tarawneh \\ Department of Mathematics, Faculty of Science, Zarqa University, Jordan
}

Copyright (C2016 by authors, all rights reserved. Authors agree that this article remains permanently open access under the terms of the Creative Commons Attribution License 4.0 International License.

\begin{abstract}
New exact solutions of the Fractional Riccati Differential equation $y^{(\alpha)}=a(x) y^{2}+b(x) y+c(x)$ are presented. Exact solutions are obtained using several methods, firstly by reducing it to second order linear ordinary differential equation, secondly by transforming it to the Bernoulli equation, finally the solution is obtained by assuming an integral condition on $\mathrm{c}$ (x) involves an arbitrary function. Using the conditions imposed on Riccati equation's coefficients we choose the form of the coefficients of the Riccati equation. For this case the general solution of the Riccati equation is also presented.
\end{abstract}

Keywords Fractional Riccati Equation, Integrability Condition, Exact Solutions

\section{Introduction}

Riccati differential equation, named after the Italian Nobleman Count Jacopo Francesco Riccati (1676-1754), it is a first order nonlinear ordinary differential equation that plays important role in mathematics and physics, as examples the motion of a particle under the influence of a power law central potential. And the application in classical mechanics is given by

$$
\frac{d y}{d x}=a(x) y^{2}+b(x) y+c(x)
$$

where a, b, c are arbitrary real functions of $\mathrm{x}$, with $\mathrm{a}, \mathrm{b}, \mathrm{c} \in \mathrm{C}$

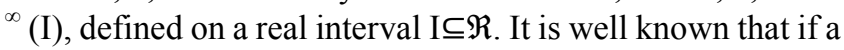
particular solution $y_{P}$ of Riccati differential equation (1) is given then its general solution takes the form

$$
y(x)=\frac{e^{\int^{x} b(z)+2 a(z) y_{P}(z) d z}}{A-\int^{x} a(z) e^{\int^{z} b(\emptyset)+2 a(\emptyset) y_{P}(\emptyset) d \emptyset} d z}+y_{P}(x)
$$

Even when a particular solution is not known Riccati differential equation (1) can be integrated if its coefficients satisfy some conditions. For example if

$$
a(x)+b(x)+c(x)=0
$$

then Riccati equation (1) has a solution given by

$$
y(x)=\frac{A+\int(a(x)+c(x)) e(x)-e(x)}{A+\int(a(x)+c(x)) e(x)+e(x)}
$$

where $e(x)=\exp \left(\int(a(x)-c(x)) d x\right)$ and $\mathrm{A}$ is an arbitrary constant [1].

On another hand the transformation $y=\frac{-u \prime}{u a(x)}$ leads to the second order linear ordinary differential equation

$$
\mathrm{u}^{\prime \prime}-\left(\frac{\mathrm{a}^{\prime}(\mathrm{x})}{a(x)}+\mathrm{b}(\mathrm{x})\right) \mathrm{u}^{\prime}+\mathrm{a}(\mathrm{x}) \mathrm{c}(\mathrm{x}) \mathrm{u}=0 .
$$

If a particular solution $y_{P}$ is known, then a general solution containing an arbitrary constant can be obtained from

$$
\mathrm{y}=\mathrm{y}_{\mathrm{P}}+\frac{1}{\mathrm{v}(\mathrm{x})} \text { [2] where } v(x) \text { is a solution of the first }
$$
order differential equation

$$
v^{\prime}=-\left(b(x)+2 a(x) w_{P}(x)\right) v-a(x)
$$

The fractional Riccati equation has the form

$$
y^{(\alpha)}=a(x) y^{2}+b(x) y+c(x)
$$

where $y^{(\alpha)}$ is the conformable fractional derivative of order $\alpha \in(0,1]$, we should remark that the methods can be generalized to include any $\alpha$. Since it is so difficult to obtain an analytical solution to Riccati differential equation One has to use a numerical method $[3,4,5,6,7,8]$ or he has to use an approximation method.

In the present work we obtained analytical solutions to Fractional Riccati equation by transforming it to Bernoulli equation, reducing it to second order linear ordinary differential equation and by considering relations among the coefficients of the Riccati equation involve some integral or differential representations, as well as the presence of some arbitrary functions.

\section{Preliminaries and Notations}

In this section, we give some basic definitions and properties of fractional calculus theory which are further used in this paper.

Definition 1 The Riemann-Liouville fractional integral operator of order $\alpha>0$, of a function $f \in C \mu, \mu \geq-1$, is defined as 


$$
{ }_{a} D_{t}^{\alpha} f(t)=\frac{1}{\Gamma(n-\alpha)}\left(\frac{d}{d t}\right)^{n} \int_{a}^{t} \frac{f(x)}{(t-x)^{\alpha-n+1}} d x
$$

$(n-1) \leq \alpha<n$, where $\alpha$ is a real number and $n$ is integer

Definition 2 The fractional derivative of $f(t)$ in the Caputo sense is defined as

$$
{ }_{a}^{c} D_{t}^{\alpha} f(t)=\frac{1}{\Gamma(n-\alpha)} \int_{a}^{t} \frac{f^{(n)}(x)}{(t-x)^{\alpha+1-n}} d x
$$

$(n-1) \leq \alpha<n$, where $\alpha$ is a real number and $n$ is integer.

Khalil, et al. [8] introduced a completely new of fractional derivative of order $\alpha \in(0,1]$ which is natural and effective than previous definitions. Also he generalized this definition for any $\alpha$. However, the case $\alpha \in(0,1]$ is the most important one, and the other cases become easy when it is established.

Definition 3 Given a function: $[0, \infty) \rightarrow \mathbb{R}$. Then the (conformable fractional derivative) of $f$ of order $\alpha \in(0,1]$ is defined by [8]

$$
T_{\alpha}(f)(t)=\lim _{\varepsilon \rightarrow 0} \frac{f\left(t+\varepsilon t^{1-\alpha}\right)-f(t)}{\varepsilon}
$$

For allt $>0, \alpha \in(0.1]$, if $f$ is $\alpha$-differentiable in some $(0, \alpha) . \alpha>0$ and, $\lim _{t \rightarrow 0^{+}} f^{(\alpha)}(t)$ exists, then define $f^{(\alpha)}(0)=\lim _{t \rightarrow 0^{+}} f^{(\alpha)}(t)$ for all $t>0, \alpha \in$ $(0,1)$.

The conformable definition treats many shortages of previous definitions such as the derivative of the constant equals to zero, the product and fractional derivative rules are satisfied, also chain rule and anti-derivative rule are satisfied. In the present work, we adopt the Conformable Fractional Derivative definition.

\section{Solution Method}

Riccati equation was studied by many researchers. In this section, we evaluate the exact solution of fractional Riccati equation with known particular solution.

Theorem 3.1. (Reduction to second order equation)

The non-linear fractional Riccati equation can be transformed to a second order linear ordinary differential equation of the form:

$$
u^{\prime \prime}-\left(\frac{\alpha-1}{x}+R(x)\right) u^{\prime}+x^{\alpha-1} S(x) u=0
$$

when $a(x)$ is non-zero and differentiable, such that $\alpha \in(0,1]$, also the solution of this equation leads us to the solution.

$$
y=\frac{-U^{\prime}(x) x^{1-\alpha}}{a(x) U(x)}
$$

Proof:

Let $\quad v=y a(x)$

$$
v^{(\alpha)}=(y a(x))^{(\alpha)}=y^{(\alpha)} a(x)+y x^{1-\alpha} a^{\prime}(x)
$$

where $y^{(\alpha)}$ denotes the FRDE also by substituting $y=\frac{v}{a}$ and some algebraic steps, then:

$$
x^{1-\alpha} v^{\prime}(x)=v^{2}+b v+c a+v x^{1-\alpha} \frac{a^{\prime}}{a}
$$

Divided both sides by $x^{1-\alpha}$, then:

$$
v^{\prime}(x)=x^{\alpha-1} v^{2}+x^{\alpha-1} b v+x^{\alpha-1} c a+v \frac{a^{\prime}}{a}
$$

Combining like terms, to get:

$$
v^{\prime}(x)=x^{\alpha-1} v^{2}+\left(x^{\alpha-1} b+\frac{a \prime}{a}\right) v+x^{\alpha-1} c a
$$

Assume: $R(x)=x^{\alpha-1} b+\frac{a^{\prime}}{a}$ and $S(x)=x^{\alpha-1} c a$, to get:

$$
\begin{gathered}
v^{\prime}(x)=x^{\alpha-1} v^{2}+R(x) v+S(x) \\
\text { Let } x^{\alpha-1} v=-\frac{u^{\prime}}{u} \\
(\alpha-1) x^{\alpha-2} v+x^{\alpha-1} v^{\prime}=\frac{-u u^{\prime \prime}+\left(u^{\prime}\right)^{2}}{u^{2}} \\
(\alpha-1) x^{\alpha-2} v+x^{\alpha-1} v^{\prime}=\frac{-u^{\prime \prime}}{u}+v^{2}\left(x^{\alpha-1}\right)^{2}
\end{gathered}
$$

Divide both sides by $x^{\alpha-1}$

$$
\frac{\alpha-1}{x} v+x^{1-\alpha} \frac{u^{\prime \prime}}{u}=x^{\alpha-1} v^{2}-v^{\prime}
$$

From equation (4)

$$
\begin{gathered}
\frac{\alpha-1}{x} v+x^{1-\alpha} \frac{u^{\prime \prime}}{u}=-\left(x^{\alpha-1} b+\frac{a^{\prime}}{a}\right) v-x^{\alpha-1} c a \\
\frac{\alpha-1}{x} v+x^{1-\alpha} \frac{u^{\prime \prime}}{u}=-R(x) v-S(x)
\end{gathered}
$$

combining like terms to get:

$$
x^{1-\alpha} \frac{u^{\prime \prime}}{u}+\left(\frac{\alpha-1}{x}+R(x)\right) v+S(x)=0
$$

divide both sides by $x^{1-\alpha}$ after substitute $v=-\frac{u \prime}{u} x^{1-\alpha}$

$$
\begin{gathered}
x^{1-\alpha} \frac{u^{\prime \prime}}{u}+\left(\frac{\alpha-1}{x}+R(x)\right)\left(-\frac{u^{\prime}}{u} x^{1-\alpha}\right)+S(x)=0 \\
\frac{u^{\prime \prime}}{u}-\left(\frac{\alpha-1}{x}+R(x)\right) \frac{u^{\prime}}{u}+x^{\alpha-1} S(x)=0 \\
\therefore u^{\prime \prime}-\left(\frac{\alpha-1}{x}+R(x)\right) u^{\prime}+x^{\alpha-1} S(x) u=0
\end{gathered}
$$

An answer of this equation will lead us to

$$
y=\frac{v}{a}=\frac{-u^{\prime} x^{1-\alpha}}{u a}
$$

Theorem 2.2. (Transform FRDE to the Bernoulli equation)

For non-linear fractional Riccati equation the substitution $v(x)=y(x)-y_{1}(x)$ will transform the (FRDE) into Bernoulli equation (ordinary differential equation of the first order), where $y_{1}$ is a known particular solution, 
Proof:

Differentiating the assumption to order $\alpha$ we get

$$
y^{(\alpha)}(x)=v^{(\alpha)}(x)+y_{1}^{(\alpha)}(x)
$$

Since $y_{1}(x)$ solves the (FRDE), it must be that

$$
y_{1}{ }^{(\alpha)}=a(x) y_{1}{ }^{2}+b(x) y_{1}+c(x)
$$

Substitute in (2)

$$
\begin{gathered}
\underbrace{v^{(\alpha)}(x)+y_{1}{ }^{(\alpha)}(x)}_{y^{(\alpha)}(x)} \\
=a(x) \underbrace{\left[v+y_{1}\right]^{2}}_{y(x)}+b(x) \underbrace{\left[v+y_{1}\right]}_{y(x)}+c(x) \\
\underbrace{x^{1-\alpha} v^{\prime}(x)}_{v^{(\alpha)}(x)}+a y_{1}{ }^{2}+b y_{1}+c \\
=a v^{2}+2 a v y_{1}+a y_{1}{ }^{2}+b v+b y_{1}+c \\
v^{\prime}(x)=a x^{\alpha-\alpha} v^{\prime}(x)=a v^{2}(x)+2 a y_{1} v(x)+b v(x) \\
v^{\prime}(x)+2 a x^{\alpha-1} y_{1} v(x)+b x^{\alpha-1} v(x) \\
\underbrace{a x^{\alpha-1}}_{q(x)} \underbrace{2}(x)
\end{gathered}
$$

This equation is of the form of Bernoulli equation with $\mathrm{n}=2$ which could be transformed to a first order linear differential equation by substituting $u=v^{-1}(x)$.

Differentiating with respect to $\mathrm{x}$ we get

$$
\frac{d u}{d x}=-v^{-2}(x) \frac{d v}{d x}
$$

Multiply (6) by $-v(x)^{-2}$

$$
\begin{aligned}
& -v^{-2} v^{\prime}+\left[2 x^{\alpha-1} a y_{1}+x^{\alpha-1} b\right] v^{-2} v=-a x^{\alpha-1} \\
& v^{\prime}+\left[2 x^{\alpha-1} a y_{1}+x^{\alpha-1} b\right] v=a \underbrace{x^{\alpha-1}}_{q(x)}
\end{aligned}
$$

The general solution is given by

$v=\frac{\int \mu(x) q(x) \cdot d x+c(x)}{\mu(x)}$

where $\mu(x)=e^{\left(\int\left[2 x^{\alpha-1} a y_{1}+x^{\alpha-1} b\right] d x\right)}$

Theorem 2.3. (Obtaining solution of FRDE by Abel's formula)

Let $y_{1}$ be a solution of (2), and assume that $z=\frac{1}{y-y_{1}}$, then the solution of FRDE is

$$
z=e^{-I\left(2 a y_{1}+b\right)} I_{\alpha}\left(e^{I\left(2 a y_{1}+b\right)}(-a(x))\right)
$$

Proof: suppose that $y_{1}$ is a solution of FRDE (2), and let $z=\frac{1}{y-y_{1}}$, then

$$
\begin{aligned}
& z\left(y-y_{1}\right)=1 \\
& y=\frac{1}{z}+y_{1}
\end{aligned}
$$

Apply $\alpha$-derivative definition to both sides of

$$
\begin{gathered}
T_{\alpha} y=T_{\alpha}\left(\frac{1}{z}\right)+T_{\alpha} y_{1} \\
T_{\alpha} y=-z^{-1-\alpha} z^{\prime}+T_{\alpha} y_{1}
\end{gathered}
$$

Substituting in the original FRDE

$$
\begin{gathered}
-z^{-1-\alpha} z^{\prime}+T_{\alpha} y_{1}=a\left[\frac{1}{z}+y_{1}\right]^{2}+b\left[\frac{1}{z}+y_{1}\right]+c \\
-z^{-1-\alpha} z^{\prime}=a\left[\frac{1}{z^{2}}+\frac{2 y_{1}}{z}+y_{1}^{2}\right]+b\left[\frac{1}{z}+y_{1}\right]+c-T_{\alpha} y_{1}
\end{gathered}
$$

but $y_{1}$ satisfies the FRDE so,

$$
\begin{aligned}
-z^{-1-\alpha} z^{\prime}= & \frac{a}{z^{2}}+\frac{2 y_{1} a}{z}+a y_{1}{ }^{2}+\frac{b}{z}+b y_{1}+c-a y^{2} \\
-b y_{1}-c &
\end{aligned}
$$

Combining like terms and divide both sides by $-z^{-1-\alpha}$, we get

$$
\begin{gathered}
z^{\prime}=-\left(2 a y_{1}+b\right) z^{\alpha}-a z^{\alpha-1}, \text { then } \\
z^{\prime}+\left(2 a y_{1}+b\right) z^{\alpha}=-a z^{\alpha-1}
\end{gathered}
$$

Multiply both sides of equation (9) by $z^{1-\alpha}$

$$
z^{1-\alpha} z^{\prime}+\left(2 a y_{1}+b\right) z=-a
$$

So, $z^{(\alpha)}+\left(2 a y_{1}+b\right) z=-a$

which is Abel's formula as we mentioned in the previous chapter.

Thus, the solution is given by

$$
z=e^{-I\left(2 a y_{1}+b\right)} I_{\alpha}\left(e^{I\left(2 a y_{1}+b\right)}(-a(x))\right) .
$$

Fractional Riccati differential equation can be considered as a quadratic equation in $\mathrm{y}(\mathrm{x})$,

$$
a(x) y^{2}+b(x) y+c(x)-y^{(\alpha)}=0
$$

then the particular solution takes the form

$y_{P}(x)=\frac{-b(x) \pm \sqrt{b^{2}(x)-4 a(x) c(x)+4 a(x) y^{(\alpha)}}}{2 a(x)}$

Analytical solutions of Riccati equation with coefficients satisfying some integral conditions or differential conditions was given by Harko et al [9]. In the following theorem we give an analytical solution of Riccati equation with coefficients satisfying an integral condition with arbitrary function.

Theorem 2.4. Assume that the coefficients of the fractional Ricatii (2), satisfies the integral condition

$$
c(x)=\frac{f_{1}(x)-\left\{b(x)+a(x)\left[\int^{x f_{1}(\phi)-b^{2}(\phi)}-A_{1}\right]\right\}^{2}}{4 a}
$$

where $A_{1}$ is an arbitrary constant of integration.

and $f_{1}$ is a new generating function satisfying the differential condition given by:

$$
b^{2}(x)+4 a(x) x^{1-\alpha} \frac{d y_{p}}{d x}=f_{1}(x)
$$

Then the general solution is given by:

$$
\begin{array}{r}
y(x)= \\
\frac{1}{e^{-I\left(2 a y_{1}+b\right)} I_{\alpha}\left(e^{I\left(2 a y_{1}+b\right)}(-a(x))\right)}+\frac{1}{2}\left[\int^{x} \frac{f_{1}(\phi)-b^{2}(\phi)}{2 a(\phi)} d \phi-A_{1}\right]
\end{array}
$$

Proof.

Assume that the arbitrary function $b(x), a(x)$ and $f_{1}(x)$ satisfying (10) then the particular solution 


$$
\begin{aligned}
& y_{p^{ \pm}}(x)=\frac{-b \pm \sqrt{f_{1}-4 a c}}{2 a} \\
& =\frac{-b \pm \sqrt{f_{1}-4 a \frac{f_{1}(x)-\left\{b(x)+a(x)\left[\int^{\left.\left.x \frac{f_{1}(\phi)-b(\phi)}{2 a(\phi)}-A_{1}\right]\right\}^{2}}\right.\right.}{4 a}}}{2 A} \\
& =\frac{-b \pm \sqrt{f_{1}-f_{1}(x)-\left\{b(x)+a(x)\left[\int \frac{x f_{1}(\phi)-b^{2}(\phi)}{2 a(\phi)}-A_{1}\right]\right\}^{2}}}{2 a}
\end{aligned}
$$

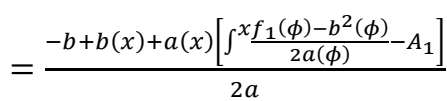

$$
\begin{aligned}
& =\frac{a(x)\left[\int^{\left.x \frac{f_{1}(\phi)-b^{2}(\phi)}{2 a(\phi)}-A_{1}\right]}\right.}{2 a} \\
& =\frac{1}{2}\left[\int \frac{x f_{1}(\phi)-b^{2}(\phi)}{2 a(\phi)}-A_{1}\right]
\end{aligned}
$$

Thus

$$
y_{p^{ \pm}}(x)=\frac{-b \pm \sqrt{f_{1}-4 a c}}{2 a}=\frac{1}{2}\left[\int \frac{x}{\left.\frac{f_{1}(\phi)-b^{2}(\phi)}{2 a(\phi)}-A_{1}\right]}\right.
$$

Then the general solution of fractional Riccati differential equation is given by

$$
\begin{array}{r}
y(x)= \\
\frac{1}{e^{-I\left(2 a y_{P}+b\right)} I_{\alpha}\left(e^{I\left(2 a y_{P}+b\right)}(-a(x))\right)}+\frac{1}{2}\left[\int^{x} \frac{f_{1}(\phi)-b^{2}(\phi)}{2 a(\phi)} d \phi-A_{1}\right]
\end{array}
$$

\subsection{Applications}

Example: - find the solution of

$$
y^{\left(\frac{1}{2}\right)}=(y-2 \sqrt{x})^{2}+1 ; y(0)=1
$$

provided that $y_{1}(x)=2 \sqrt{x}$ is a solution.

Solution: It is to verify that $y_{1}=2 \sqrt{x}$ is a solution to equation (12).

Make the change of variables by Substituting $y=v+$ $2 \sqrt{x}$ and $y^{\left(\frac{1}{2}\right)}=v^{\left(\frac{1}{2}\right)}+1$ yields

$$
v^{\left(\frac{1}{2}\right)}+1=(v+2 \sqrt{x}-2 \sqrt{x})^{2}+1
$$

Which can be simplify to a Bernoulli equation for $v$

$$
v^{\prime}=x^{-\frac{1}{2}} v^{2}
$$

Let $u=v^{-1}$

$$
u^{\prime}=-v^{-2} v^{\prime}
$$

Multiply equation (13) by $-v^{-2}$

$$
\begin{gathered}
-v^{-2} v^{\prime}=-x^{-\frac{1}{2} v^{-2} v^{2}} \\
u^{\prime}=-x^{-\frac{1}{2}}=\frac{-1}{\sqrt{x}} \\
\frac{d u}{d x}=\frac{-1}{\sqrt{x}} \rightarrow d u=\frac{-1}{\sqrt{x}} d x \\
u=\int \frac{-1}{\sqrt{x}} \cdot d x=-2 \sqrt{x}+c \\
\frac{1}{v}=-2 \sqrt{x}+c
\end{gathered}
$$

$$
v=\frac{1}{-2 \sqrt{x}+c}
$$

but $y=v+2 \sqrt{x}$ then

$$
y=\frac{1}{-2 \sqrt{x}+c}-2 \sqrt{x}
$$

the initial condition $y(0)=1$ implies that $\quad c=1$ so, the general solution is given by

$$
y=\frac{3}{-4 \sqrt{x^{3}}+1}-\frac{x^{2}}{2}
$$

\section{Conclusions}

In this thesis we found an exact solution of the fractional Riccati differential equation using the conformable fractional derivative which is simpler and more efficient. The new definition reflects a natural extension of normal derivative to solve fractional differential equation.Also we introduced some theorems which lead us to find a second solution when a particular solution was given.

\section{REFERENCES}

[1] E. Kamke, Differential gleichungen: L“osungsmethoden und L"osungen, Chelsea, New York (1959).

[2] Weisstein, E. W.; Riccati Differential Equation; From MathWorld-A Wolfram WebResource.http://mathworld.wol fram.com/RiccatiDifferentialEquation.html.

[3] He, J. H. (1999), Variational Iteration Method-a Kind Of Non-Linear Analytical Technique: Some Examples, International Journal of Non-Linear Mechanics, 34: 699-708.

[4] Khader, M. M., EL Danaf, T. S. and Hendy, A. S. (2012), Efficient Spectral Collocation Method for Solving Multi-Term Fractional Differential Equations Based on the Generalized Laguerre Polynomials, Journal of Fractional Calculus and Applications, 3(13), 1-14.

[5] Momani, Shaher and Shawagfeh, Nabil (2006), Decomposition Method for Solving Fractional Riccati Differential Equations, Applied Mathematics and Computation, 182(2): 1083-1092.

[6] Odibat, Zaid and Momani, Shaher (2008), Modified Homotopy Perturbation Method: Application to Quadratic Riccati Differential Equation of Fractional Order, Chaos, Solutions \& Fractals, 36(1), 167-174.

[7] Wu, Fei and Huang, Lan. Lan (2014), Approximate Solutions of Fractional Riccati Equations using the Adomain decomposition Method, Abstract and Applied

[8] Khalil, Roshdi, Horani, M., Yousef, Abdelrahman and Sababheh, M. (2014), A New Definition of Fractional Derivative, Computational and Applied Mathematics, 246: 65-70.

[9] Harko, T., Lobo, F. and Mak, M. K. (2013), Analytical Solutions of the Riccati Equation with Coefficients Satisfying Integral Or Differential Conditions With Arbitrary Functions, Universal Journal of Applied Mathematics, 2: 109-118. 